\title{
Efeitos de um programa de exercício aeróbio nos níveis de atividade física em pacientes hemodialisados
}

\author{
Effects of aerobic exercise program on physical activity levels in \\ hemodialysis patients
}

\author{
José Florêncio Sousa, ${ }^{1 *}$ Jonatas Cassiano Ribeiro, ${ }^{1}$ Carla Correia Sá, ${ }^{1,2}$ André Novo, ${ }^{2,3}$ \\ Vítor Pires Lopes ${ }^{1,2}$
}

ARTIGO ORIGINAL | ORIGINAL ARTICLE

\begin{abstract}
RESUMO
O estudo teve como objetivo avaliar o efeito de um programa de exercício físico nos níveis de atividade física (AF) habitual em pacientes hemodialisados. A amostra foi composta por 24 pacientes hemodialisados, dos quais $45.8 \%$ do sexo feminino, com uma idade média de $75.1 \pm 11.8$ anos, que participaram num programa de exercício aeróbio intradialítico, 3 vezes por semana, ao longo de 8 semanas. A carga de treino foi individualizada, com aumento progressivo de acordo com a autoperceção de esforço. Foram utilizados acelerómetros para a avaliação da AF durante uma semana completa, tanto antes como depois da aplicação do programa. Os resultados indicaram que a AF predominantemente realiza$\mathrm{da}$, em ambos os momentos de avaliação, foi de intensidade leve. Por outro lado, a AF moderada e vigorosa juntas perfizeram menos de $1 \%$ do total de AF realizada. O tempo despendido em AF leve aumentou significativamente entre o pré e o pós-teste $(p=0.03)$, enquanto o tempo despendido em atividades sedentárias, AF moderada e AF vigorosa não se alterou de forma significativa. Em conclusão, os níveis de AF leve aumentaram de forma significativa entre o pré e o pós-teste, não se registando mudanças significativas no tempo de sedentarismo, na AF moderada e na AF vigorosa.

Palavras-chave: acelerómetros, treino físico, insuficiência renal cronica, idosos
\end{abstract}

ABSTRACT

The purpose of this study was to evaluate the effectiveness of an exercise program on changes in physical activity (PA) levels of hemodialysis patients. The sample consisted of 24 hemodialysis patients, $45.8 \%$ of female subjects, with an average age of $75.1 \pm 11.8$ years, were subjected to an aerobic exercise program intradialytic 3 times per week over 8 weeks. The training load was individualized with progressive increase through the self-perception effort. Accelerometers were used for the evaluation of PA during a continuous week, before and after the implementation of the program. The results suggested that PA mainly performed in both moments of evaluation was light intensity, on the other hand, the moderate and vigorous PA together constituted less than $1 \%$ of total PA performed. The time spent in light PA increased significantly between the pre and post-test $(p=0.03)$, while the time spent in sedentary activities, moderate PA and vigorous PA did not change significantly. In conclusion, light PA significantly increased between the pre and post-test. There were not significant changes in sedentary time, in moderate and vigorous PA.

Keywords: accelerometers, physical training, chronic renal failure, elderly

Artigo recebido a 14.06.2013; $1^{\text {a }}$ Revisão 18.09.2013; 2 ${ }^{\text {a }}$ Revisão 03.10.2013; Aceite 15.10.2013

${ }^{1}$ Departamento de Ciências do Desporto e Educação Física, Instituto Politécnico de Bragança, Bragança, Portugal

${ }^{2}$ Centro de Investigação em Desporto, Saúde e Desenvolvimento Humano (CIDESD), Vila Real, Portugal

${ }^{3}$ Departamento de Ciências de Enfermagem, Instituto Politécnico de Bragança, Bragança, Portugal

* Autor correspondente: Departamento de Ciências do Desporto e Educação Física, Instituto Politécnico de Bragança, Campus de Santa Apolónia, 5301-856 Bragança - Portugal; E-mail: florencio.flor@ hotmail.com 


\section{INTRODUÇÃO}

O número de doentes renais crónicos em estádio terminal tem vindo a crescer nos últimos anos (United States Renal Data System [USRDS], 2012) e em 2004 existiam mais de 1371000 pessoas a recorrerem à diálise em todo o mundo e cerca de 324000 na Europa (Grassmann, Gioberge, Moeller, \& Brown, 2005). O aumento da prevalência da diabetes mellitus e da hipertensão arterial, em conjunto com a melhoria dos tratamentos médicos e do aumento da esperança média de vida explica em parte o aumento dos casos de pessoas com esta síndrome (Coresh et al., 2007). O recente relatório anual do gabinete de registo do tratamento da doença renal terminal (Macário, 2013) revela que em 2012 em Portugal existiam 11282 pacientes em diálise $(93.4 \%$ em hemodiálise), o que significa um crescimento de $16.6 \%$ relativamente ao ano de 2007.

Os pacientes hemodialisados apresentam um risco acrescido de morte por doença cardiovascular até 50 vezes maior em relação à população em geral (Kundhal \& Lok, 2005), o que está em grande parte associado com o aumento de outras comorbilidades que geralmente afetam os doentes renais crónicos em estádio terminal, como é o caso da hipertensão e da aterosclerose. Para além destas patologias, os pacientes hemodialisados apresentam uma aptidão física diminuída, em cerca de $60 \%$ a $70 \%$ do que era esperado para a sua idade, em relação aos indivíduos saudáveis (Painter, 2005).

A insuficiência renal crónica, em conjunto com as várias patologias que lhe estão associadas e a reduzida aptidão física explicam em grande parte os motivos pelos quais os pacientes hemodialisados apresentam níveis tão baixos de atividade física (AF) (Avesani et al., 2012). Existem dados que demonstram que os pacientes hemodialisados têm um nível de $\mathrm{AF}$ significativamente mais baixo relativamente aos indivíduos saudáveis sedentários (Johansen et al., 2000), levando a pensar que um paciente hemodialisado de 30 anos possa ter menos AF diária que um indivíduo sedentário saudável de
70 anos (Ikizler \& Himmelfarb, 2006). Tal como acontece na população em geral, os níveis de AF nos pacientes hemodialisados estão estritamente relacionados com a taxa de mortalidade (Stack, Molony, Rives, Tyson, \& Murthy, 2005). Como forma de reverter esta situação, tem-se proposto a aplicação de programas de exercício físico antes/durante a hemodiálise (Clyne, 2011). Recentemente surgiram várias investigações que comprovam a eficácia destes programas no aumento da AF, da aptidão física e na melhoria do quadro clínico de algumas patologias (Segura-Orti \& Johansen, 2010), no entanto ainda não é claro que estas alterações permaneçam após o fim destes programas.

Este estudo tem como objetivo avaliar a eficácia de um programa de exercício físico nas alterações dos níveis de AF dos pacientes hemodialisados.

\section{MÉTODO}

Estudo quasi-experimental, tipo ensaio clínico não controlado.

\section{Participantes}

Para a formação da amostra foram analisados todos os pacientes, que realizavam o tratamento de hemodiálise numa clínica localizada no nordeste de Portugal. Todos os pacientes da clínica foram potenciais participantes no estudo, contudo foram excluídos os sujeitos que apresentavam algum dos critérios de exclusão referidos na Tabela 1. Após aplicados os critérios de exclusão resultou um grupo com condições para participar no programa de intervenção, do qual foram selecionados por conveniência 24 indivíduos. A média de idades da amostra era de $75.1 \pm 11.8$ anos, sendo constituída por $45.8 \%$ indivíduos do sexo feminino. Em média, os pacientes hemodialisados realizavam o tratamento de hemodiálise há $5.1 \pm 3.5$ anos, e na sua maioria, apresentavam outras patologias das quais se destacam a hipertensão arterial $(21.7 \%)$ e a diabetes mellitus $(13.0 \%)$, contudo controladas. Todos os participantes do estudo realizavam avaliações 
cardiológicas de 6 em 6 meses e em todas as sessões foram acompanhadas por um médico especialista em saúde geral e familiar e por um médico nefrologista. Nenhum dos pacientes que participou no estudo apresentava contraindicações relativamente à participação neste tipo de programa.

Antes e após uma semana do programa de exercício foi realizada a avaliação da AF (diária), da massa corporal e da estatura.

Tabela 1

Critérios de exclusão

- Menos de três meses de tratamento de hemodiálise ininterrupto

- Hipertensão arterial não controlada

- Angina instável

- Diabetes mellitus não controlada

- Transtorno cognitivo incapacitante para executar ordens

- Cirurgia recente sem alta médica

- Insuficiência cerebrovascular com síncopes recidivantes

- Insuficiência cardíaca com escala de New York Heart Association três ou mais

\section{Instrumentos}

Avaliação da atividade física

Para avaliar a AF utilizou-se o monitor de AF Actigraph ${ }^{\circledR}$ modelo GT3X (ActiGraph ${ }^{\circledR}$ Pensacola, FL, Estados Unidos da América), que é um instrumento que avalia de forma objetiva a $\mathrm{AF}$, que não necessita de ser calibrado antes de ser utilizado. Este acelerómetro triaxial regista as acelerações realizadas nos três planos de movimento, transformando estas acelerações em counts, que foram guardados em epochs de 1 minuto, o que resulta nas contagens por minuto (cpm). Os acelerómetros foram colocados junto à cintura do paciente hemodialisado durante 7 dias consecutivos. Foram dadas indicações para só retirar o instrumento quando fosse dormir, tomar banho e quando realizasse atividades no meio aquático. Os dados das epochs armazenados no acelerómetro foram posteriormente analisados pelo software Actilife 6 disponibilizado pela Acti-
Graph $^{\circledR}$. Os dados dos acelerómetros só foram considerados válidos se estes fossem usados em pelo menos 5 dos 7 dias de avaliação e só foram considerados válidos os dias em que o acelerómetro fosse utilizado pelo menos 10 horas por dia. Os períodos superiores a 60 minutos com contínuos zeros, com tolerância para 2 minutos de counts entre 0 e 100 foram considerados como períodos de não utilização do acelerómetro. Através das cpm foram criados pontos de corte, que representam diferentes equivalentes metabólicos (MET's). Os pontos de corte utilizados neste estudo baseiam-se nos propostos pelo estudo de Sasaki, John e Freedson (2011), aos quais foram ainda adicionados os pontos de corte para atividade sedentária. No presente estudo foram aplicados os seguintes pontos de corte: 0-99 cpm atividade sedentária; $100-2689 \mathrm{cpm}$ - AF leve; 2690-6166 cpm - AF moderada; 6167-9642 cpm - AF vigorosa; e $\geq 9642 \mathrm{cpm}$ - AF muito vigorosa. O tempo despendido nos diferentes níveis de AF foi expresso em minutos.

\section{Avaliação antropométrica}

A estatura foi avaliada através do estadiómetro portátil Invicta ${ }^{\circledR}$, modelo Leicester (Birmingham, Inglaterra). Para a realização da medição da estatura, foi pedido ao paciente que permanecesse na posição anatómica, com os pés descalços sobre a base do estadiómetro e a cabeça posicionada no plano horizontal de Frankfurt. A estatura registada resultou da média de duas avaliações consecutivas. Para avaliação da massa corporal foi utilizada a balança portátil Seca ${ }^{\circledR}$, modelo M889, (Hamburgo, Alemanha), em que o paciente tinha de permanecer imóvel em cima da balança, descalço e com roupas leves. O IMC foi calculado através da divisão da massa corporal $(\mathrm{kg})$ pelo quadrado da estatura $\left(\mathrm{m}^{2}\right)$.

\section{Procedimentos}

Foi aplicado um programa de exercício físico ao longo de 8 semanas consecutivas, numa clínica de hemodiálise a pacientes com insuficiência renal crónica. O programa consistiu em 
exercício aeróbio, realizados 3 vezes por semana. O exercício era realizado durante as sessões de hemodiálise e consistia em pedalar num cicloergómetro horizontal Carex ${ }^{\circledR}$ Digital Pedal Exerciser (Sioux Falls, SD, Estados Unidos da América). O período de treino decorria durante a hemodiálise, depois dos primeiros 30 e antes do último dos 45 minutos de hemodiálise, altura em que se verifica menor variabilidade cardíaca durante o tratamento. A carga de treino foi individualizada com aumento progressivo, mediante autoperceção do esforço avaliado com a escala de Borg (Borg, 1982), à qual os participantes tinham sido previamente familiarizados. O programa de exercício físico foi constantemente supervisionado e monitorizado por duas fisioterapeutas, que durante o período de treino apenas tinham esta função e foram sistematicamente acompanhados pelos médicos e enfermeiros da clínica, que monitorizaram o tratamento por hemodiálise.

$\mathrm{Na}$ primeira semana ajustou-se o grau de resistência do cicloergómetro, de modo a que o paciente hemodialisado conseguisse realizar o exercício de forma constante, na máxima intensidade possível que lhe permitisse manter essa mesma intensidade de exercício durante 25 minutos. Na segunda semana manteve-se o mesmo grau de resistência do cicloergómetro e acrescentou-se 5 minutos à duração da sessão. $\mathrm{Na}$ terceira semana voltou-se a aumentar a resistência do cicloergómetro, de modo a que o paciente hemodialisado conseguisse realizar $o$ exercício de forma constante, na máxima intensidade possível que lhe permitisse manter essa mesma intensidade de exercício com a mesma duração da semana anterior. Na quarta semana manteve-se o mesmo grau de resistência do cicloergómetro relativamente à semana anterior e acrescentou-se 5 minutos à duração da sessão. Este modo de progressão da carga manteve-se até ao fim do programa de intervenção.

O estudo seguiu todos os princípios da declaração de Helsínquia. Todos os pacientes hemodialisados assinaram o consentimento informado relativo à sua disponibilidade para participação neste estudo.

\section{Análise Estatística}

Foi analisada a normalidade das distribuições das variáveis através do teste de ShapiroWilk com correção de Liliefors. Apenas o tempo despendido em AF moderada e vigorosa não apresentaram uma distribuição normal. Para a análise das diferenças entre o pré e o pós-teste do tempo despendido em AF sedentária e leve recorreu-se ao teste $t$-Student para amostras emparelhadas. Para análise das diferenças entre pré e pós-teste do tempo despendido em $\mathrm{AF}$ moderada e vigorosa recorreu-se ao teste nãoparamétrico de Wilcoxon. O nível significância foi fixado para $p<0.05$. Toda a análise estatística foi realizada através do SPSS versão 21 .

\section{RESULTADOS}

Antes do programa de intervenção, os pacientes hemodialisados tinham um índice de massa corporal médio de $24.3 \pm 2.6 \mathrm{~kg} / \mathrm{m}^{2}$, não se registando mudanças significativas neste parâmetro após o programa de exercício ( $p=$ $0.44)$. Os pacientes hemodialisados não realizaram nenhuma AF muito vigorosa em ambos os momentos de avaliação. A AF predominantemente realizada pelos pacientes hemodialisados, quer antes, quer depois do programa de intervenção era de intensidade leve e o tempo despendido em AF moderada e vigorosa juntas perfaziam, quer no pré-teste quer no pós-teste, menos de $1 \%$ do total de AF realizada pelos pacientes hemodialisados (Tabela 2).

Os resultados presentes na Tabela 2 indicam que entre o pré e o pós-teste existiu um aumento não significativo do tempo despendido em AF sedentária $(p=0.08)$ e do tempo despendido em AF vigorosa $(p=0.15)$. O tempo despendido em AF moderada apresentou uma diminuição entre pré e o pós-teste, mas sem significância estatística $(p=0.37)$. O único parâmetro que apresentou alterações significativas entre os dois momentos de avaliação foi o tempo dependido em AF leve, que aumentou entre o pré e o pós-teste $(p=0.03)$. 
Tabela 2

Resultados do pré e pós-teste, expressos em média e desvio padrão para o tempo despendido em AF sedentária e leve, e a mediana e o intervalo interquartil para o tempo despendido em AF moderada e vigorosa

\begin{tabular}{ccc}
\hline & Pré-Teste & Pós-Teste \\
\hline Atividade física sedentária (min) & $408.95 \pm 182.77$ & $477.68 \pm 183.17$ \\
Atividade física leve (min) & $423.76 \pm 187.12$ & $493.87 \pm 174.26^{*}$ \\
Atividade física moderada (min) & $2.28 \pm 8.37$ & $1.43 \pm 4.70$ \\
Atividade física vigorosa (min) & $0.00 \pm 0.13$ & $0.13 \pm 0.14$ \\
\hline Nota $^{*} p<0.05$ & &
\end{tabular}

\section{DISCUSSÃO}

O principal objetivo deste estudo foi avaliar o impacto de um programa de exercício físico na mudança dos níveis de AF. Os resultados demonstram que existiu um aumento significativo do tempo despendido na AF leve entre o pré e o pós-teste e não se registaram mudanças significativas no tempo de sedentarismo, na AF moderada e na AF vigorosa.

Uma das principais barreiras para a prática de AF vulgarmente mencionada pelos pacientes hemodialisados é a elevada fadiga que esta provoca (Delgado \& Johansen, 2012), existindo mesmo várias indicações que apontam a sensação de fadiga como o fator mais importante que influencia os baixos níveis de AF dos pacientes hemodialisados (Mahrova \& Svagrova, 2013). O tratamento de hemodiálise aumenta os níveis de fadiga (Lobbedez et al., 2008), podendo os pacientes hemodialisados apresentar três vezes mais fadiga em comparação com a população adulta saudável (Johansen, Doyle, Sakkas, \& Kent-Braun, 2005). Esta elevada prevalência de fadiga nos pacientes hemodialisados resulta de um conjunto de fatores inerentes a esta patologia, das quais se pode destacar a forte deterioração dos níveis de aptidão física. Existe alguma evidência de que o consumo máximo de oxigénio $\left(\mathrm{VO}_{2} \mathrm{máx}\right) \mathrm{em}$ pacientes hemodialisados geralmente se situa entre $50 \%$ a $80 \%$ do verificado na população saudável, rondado valores entre 15 a 21 $\mathrm{ml} / \mathrm{kg} / \mathrm{min}$ (Johansen \& Painter, 2012). Este tipo de pacientes para além de terem uma condição cardiorrespiratória reduzida, apresentam uma forte atrofia muscular (Kouidi et al., 1998). Existem ainda dados que indicam que esta degradação muscular é ainda mais forte nas fibras do tipo II (Kouidi et al., 1998), o que ajuda a compreender as causas inerentes à fraca capacidade de produção de força destes doentes em comparação com a população em geral (Johansen et al., 2003).

Há muitos anos que esta debilidade física dos pacientes hemodialisados está identificada como uma das causas para a elevada taxa de incapacidade para o trabalho (Gutman, Stead, \& Robinson, 1981), reduzindo também a sua capacidade na realização das tarefas quotidianas (Johansen \& Painter, 2012), tornando-os dependentes do apoio constante de terceiros (Tamura et al., 2009). Dado que o presente estudo é composto maioritariamente por pessoas idosas, é expectável que este conjunto de fragilidades e dependência esteja ainda mais agravado (Odden, 2010), o que reforça a explicação para os reduzidos níveis de AF dos sujeitos da amostra. Segundo vários investigadores (Jhamb, Weisbord, Steel, \& Unruh, 2008; Segura-Orti \& Johansen, 2010), esta cascata de influências negativas pode ser revertida através da implementação de programas de exercício físico. Prova dos possíveis benefícios do treino físico é o estudo de Chang, Cheng, Lin, Gau e Chao (2010), que avaliou o efeito de um programa de exercício aeróbio de baixa intensidade, realizado durante as sessões de hemodiálise através de ciclo ergómetro, com uma duração de 30 minutos, durante 8 semanas. Este estudo concluiu que o programa de treino conseguiu 
reduzir de forma significativa os níveis de fadiga dos pacientes hemodialisados, estimulando o aumento dos níveis de AF, estando estes dois fatores significativamente correlacionados entre si.

Este tipo de efeitos gerados pela participação em programas de exercício físico podem ajudar a explicar quais os possíveis fatores responsáveis pela mudança de comportamentos verificados no presente estudo. No fim do programa de intervenção, provavelmente o paciente hemodialisado terá uma melhor aptidão física (Cheema, Smith, \& Singh, 2005) e consequentemente menores níveis de fadiga (Bossola, Vulpio, \& Tazza, 2011), o que permitirá alcançar níveis de AF mais elevados comparativamente aos verificados antes do programa de intervenção. Esta situação pode conduzir ainda à melhoria da aptidão funcional que também está associada com a AF (Majchrzak et al., 2005). O próprio aumento da $\mathrm{AF}$ decorrente do programa de intervenção parece determinar em boa parte os níveis de fadiga dos pacientes hemodialisados (Brunier \& Graydon, 1993), ou seja, baixos níveis de AF levam a maiores níveis de fadiga e elevados níveis de AF levam a menores níveis de fadiga. Assim, é compreensível que os pacientes hemodialisados com baixos níveis de AF entrem numa espiral negativa, onde a baixa aptidão física e a elevada fadiga são responsáveis pela incapacidade do paciente hemodialisado contrariar esta situação. Assim sendo, o recurso a este tipo de programas pode contrariar essa espiral negativa.

Embora a maioria dos pacientes hemodialisados admita que o estilo de vida sedentário acarreta elevados riscos de saúde e que o aumento da AF é benéfico para o seu estado de saúde (Delgado \& Johansen, 2012), é reconhecido que a maior parte das pessoas que padecem desta síndrome não conseguem cumprir as recomendações mínimas de AF relacionadas com a saúde dos idosos, de 30 minutos de AF moderada a vigorosa por dia, 5 vezes por semana ou 20 minutos de AF vigorosa por dia, 3 vezes por semana (Painter, Ward, \& Nelson,
2011). De uma forma geral, os pacientes hemodialisados referem que esta incapacidade se deve principalmente à grande sensação de fadiga sentida quando realizam AF moderada e vigorosa (Stack et al., 2005). De facto, o nível de AF moderada e vigorosa apresentada pelos pacientes hemodialisados do presente estudo está substancialmente abaixo do nível de $\mathrm{AF}$ da população em geral. Por exemplo, em média um idoso norte-americano ( $\geq 70$ anos) realiza entre 5 a 9 minutos de AF moderada e vigorosa por dia (Troiano et al., 2008) enquanto os sujeitos do presente estudo realizam cerca de 2 minutos.

Talvez o conjunto de alterações metabólicas presentes nos pacientes hemodialisados crie uma debilidade física tão forte e persistente que o programa de exercício aeróbio não conseguiu colmatar com melhorias suficientes para reverter esta sensação de fadiga ao realizar AF moderada e vigorosa, levando a que não tivessem sido registadas alterações significativas no tempo despendido nestas intensidades entre pré e o pós-treino.

A sensação de fadiga parece dissipar-se com a diminuição da intensidade da $\mathrm{AF}$, não afetando de forma tão expressiva a realização de tarefas de baixa intensidade (Stack et al., 2005). Storer, Casaburi, Sawelson e Kopple (2005) concluíram que um programa de exercício aeróbio realizado num cicloergómetro durante 9 semanas a uma baixa intensidade consegue aumentar de forma significativa a capacidade cardiorrespiratória, força, potência e diminuir a fadiga em pacientes hemodialisados. Vários estudos (Mercer, Crawford, Gleeson, \& Naish, 2002; Painter, Carlson, Carey, Paul, \& Myll, 2000a) têm demonstrado que os pacientes hemodialisados que participam neste tipo de programas de intervenção melhoram diversas componentes da aptidão funcional, sendo este um fator importante para que estes doentes de idade avançada consigam realizar as suas tarefas da vida quotidiana. Painter, Carlson, Carey, Paul e Myll (2000b) destacam que um programa de treino para além de melhorar a aptidão funcional dos pacientes hemodialisados conse- 
gue melhorar diversas componentes da qualidade de vida. Este conjunto de melhorias, alcançadas com a prática de um programa de exercício físico, podem não ser suficientes para que os pacientes hemodialisados consigam realizar mais AF de intensidade elevada, mas podem ser a causa para o aumento significativo da AF leve registada no presente estudo.

Mesmo que os programas de exercício físico não consigam trazer melhorias expressivas na condição física dos pacientes hemodialisados, existem outros benefícios decorrentes deste tipo de programas que podem manter os níveis de AF em patamares mais elevados. O aumento da AF durante os programas de intervenção é uma mais-valia no combate aos diversos problemas de saúde que afetam os pacientes hemodialisados, como é o caso da hipertensão, resistência à insulina ou dislipidemias (Cheema \& Singh, 2005). É conhecida a elevada prevalência de diabetes mellitus nos pacientes hemodialisados (USRDS, 2012), sendo esta uma das patologias que mais influencia negativamente os níveis de AF dos pacientes hemodialisados (Avesani et al., 2012). O aumento da AF decorrente dos programas de exercício físico poderá então menorizar os efeitos negativos da diabetes mellitus e assim conseguir ao mesmo tempo estimular o aumento da AF. Os programas de exercício físico também melhoram de forma significativa as situações de depressão que costumam caracterizar os pacientes hemodialisados (Levendoglu et al., 2004), que estão fortemente afetados na sua saúde mental com o decorrer dos tratamentos de hemodiálise (Braga et al., 2011). A melhoria deste aspeto da saúde mental também está associada com a redução da sensação de fadiga (Jhamb et al., 2008), que posteriormente poderá favorecer o aumento da prática de AF.

A possível melhoria da aptidão física, da aptidão funcional, da saúde mental, da qualidade de vida e da fadiga podem ser os pilares que sustentam os mecanismos responsáveis pelo aumento significativo da AF leve registado neste estudo. O estudo de Van Heuvelen, Kempen, Ormel e Rispens (1998) demonstrou que o tempo passado em AF de lazer, incluindo a de baixa intensidade, está positivamente associado com a maior parte das componentes da aptidão física, independentemente da idade das pessoas idosas. Portanto, os pacientes hemodialisados ao aumentarem os seus níveis de AF leve podem estar a garantir que a sua aptidão física não se degrade rapidamente, criando assim um efeito benéfico recíproco, que ajudará a manter a $\mathrm{AF}$ em níveis mais elevados, quando comparado com os registados antes do programa de intervenção. Este aumento dos níveis de AF dos pacientes hemodialisados após um programa de exercício é de especial relevância, não só pela melhoria da qualidade de vida destas pessoas, mas também pela diminuição do risco de morte que estas passam a ter (Matsuzawa et al., 2012).

\section{CONCLUSÕES}

Em conclusão, o programa de exercício aeróbio aumentou de forma significativa o tempo despendido na AF leve entre o pré e o pós-teste e não se registaram mudanças significativas no tempo de sedentarismo, na $\mathrm{AF}$ moderada e na AF vigorosa.

\section{Agradecimentos:}

Os autores agradecem à Tecsam - Clínica de Hemodiálise de Mirandela pela sua disponibilidade na colaboração do estudo.

\section{Conflito de Interesses:}

Nada a declarar.

Financiamento:

Nada a declarar.

\section{REFERÊNCIAS}

Avesani, C. M., Trolonge, S., Deleaval, P., Baria, F., Mafra, D., Faxen-Irving, G., ... Fouque, D. (2012). Physical activity and energy expenditure in haemodialysis patients: An international survey. Nephrology Dialysis Transplantation, 27(6), 2430-2434. doi: 10.1093/Ndt/Gfr692 
Borg, G. A. (1982). Psychophysical bases of perceived exertion. Medicine and Science in Sports and Exercise, 14(5), 377-381.

Bossola, M., Vulpio, C., \& Tazza, L. (2011). Fatigue in chronic dialysis patients. Seminars in Dialysis, 24(5), 550-555. doi: 10.1111/j.1525-139X. 2011.00956.x

Braga, S. F., Peixoto, S. V., Gomes, I. C., Acurcio, F. A., Andrade, E. I., \& Cherchiglia, M. L. (2011). Fatores associados com a qualidade de vida relacionada à saúde de idosos em hemodiálise. Revista de Saude Publica, 45, 1127-1136. doi: 10.1590/S0034-89102011000600015

Brunier, G. M., \& Graydon, J. (1993). The influence of physical activity on fatigue in patients with ESRD on hemodialysis. American Nephrology Nurses' Association, 20(4), 457-461.

Chang, Y. M., Cheng, S. Y., Lin, M. L., Gau, F. Y., \& Chao, Y. F. C. (2010). The effectiveness of intradialytic leg ergometry exercise for improving sedentary life style and fatigue among patients with chronic kidney disease: A randomized clinical trial. International Journal of Nursing Studies, 47(11), 1383-1388. doi: 10.1016/j.ijnur stu.2010.05.002

Cheema, B. S. B., \& Singh, M. A. F. (2005). Exercise training in patients receiving maintenance hemodialysis: A systematic review of clinical trials. American Journal of Nephrology, 25(4), 352364. doi: 10.1159/000087184

Cheema, B. S. B., Smith, B. C. F., \& Singh, M. A. F. (2005). A rationale for intradialytic exercise training as standard clinical practice in ESRD. American Journal of Kidney Diseases, 45(5), 912916. doi: 10.1053/j.ajkd.2005.01.030

Clyne, N. (2011). Exercise training in chronic kidney disease. US Nephrology, 5(2), 15-17.

Coresh, J., Selvin, E., Stevens, L. A., Manzi, J., Kusek, J. W., Eggers, P., ... Levey, A. S. (2007). Prevalence of chronic kidney disease in the United States. Journal of the American Medical Association, 298, 2038-2047.

Delgado, C., \& Johansen, K. L. (2012). Barriers to exercise participation among dialysis patients. Nephrology Dialysis Transplantation, 27(3), 11521157. doi: $10.1093 / \mathrm{Ndt} / \mathrm{Gfr} 404$

Grassmann, A., Gioberge, S., Moeller, S., \& Brown, G. (2005). ESRD patients in 2004: Global overview of patient numbers, treatment modalities and associated trends. Nephrology Dialysis Transplantation, 20(12), 2587-2593.
Gutman, R. A., Stead, W. W., \& Robinson, R. R. (1981). Physical-activity and employment status of patients on maintenance dialysis. New England Journal of Medicine, 304 (6), 309-313.

Ikizler, T. A., \& Himmelfarb, J. (2006). Muscle wasting in kidney disease: Let's get physical. Journal of the American Society of Nephrology, 17, 2097-2098. doi: 10.1681/Asn.2006060629

Jhamb, M., Weisbord, S. D., Steel, J. L., \& Unruh, M. (2008). Fatigue in patients receiving maintenance dialysis: A review of definitions, measures, and contributing factors. American Journal of Kidney Diseases, 52(2), 353-365. doi: 10.1053/j.ajkd.2008.05.005

Johansen, K. L., Chertow, G. M., Ng, A. V., Mulligan, K., Carey, S., Schoenfeld, P. Y., \& KentBraun, J. A. (2000). Physical activity levels in patients on hemodialysis and healthy sedentary controls. Kidney International, 57(6), 2564-2570. doi: 10.1046/j.1523-1755.2000.00116.x

Johansen, K. L., Doyle, J., Sakkas, G. K., \& KentBraun, J. (2005). Neural and metabolic mechanisms of excessive muscle fatigue in maintenance hemodialysis patients. American Journal of Physiology, 289(3), 805-813.

Johansen, K. L., \& Painter, P. (2012). Exercise in individuals with CKD. American Journal of Kidney Diseases, 59(1), 126-134. doi: 10.1053/j.ajkd. 2011.10.008

Johansen, K. L., Shubert, T., Doyle, J., Soher, B., Sakkas, G. K., \& Kent-Braun, J. A. (2003). Muscle atrophy in patients receiving hemodialysis: Effects on muscle strength, muscle quality, and physical function. Kidney International, 63(1), 291-297.

Kouidi, E., Albani, M., Natsis, K., Megalopoulos, A., Gigis, P., Guiba-Tziampiri, O., ... Deligiannis, A. (1998). The effects of exercise training on muscle atrophy in haemodialysis patients. Nephrology Dialysis Transplantation, 13(3), 685699. doi: 10.1093/Ndt/13.3.685

Kundhal, K., \& Lok, C. E. (2005). Clinical epidemiology of cardiovascular disease in chronic kidney disease. Nephron Clinical Practice, 101(2), C47-C52. doi: 10.1159/000086221

Levendoglu, F., Altintepe, L., Okudan, N., Ugurlu, H., Gokbel, H., Tonbul, Z., ... Turk, S. (2004). A twelve week exercise program improves the psychological status, quality of life and work capacity in hemodialysis patients. Journal of Nephrology, 17(6), 826-832. 
Lobbedez, T., Desbordes, E., Joly, F., Ficheux, M., Henri, P., \& Ryckelynck, J. P. (2008). Fatigue in elderly patients on dialysis. Néphrologie $\mathcal{E}$ thérapeutique, 4(7), 584-589. doi: 10.1016/j. nephro.2008.04.007

Macário, F. (2013). Tratamento substitutivo renal da doença renal crónica estádio $V$ em Portugal. Disponível em: www.spnefro.pt/comissoes_gabine tes/Gabinete_registo_2012/registo_2012.pdf

Mahrova, A., \& Svagrova, K. (2013). Exercise therapy - Additional tool for managing physical and psychological problems on hemodialysis. $\mathrm{H}$. Suzuki (Ed.), Hemodialysis (ch. 36). Intech. doi: $10.5772 / 53058$

Majchrzak, K. M., Pupim, L. B., Chen, K., Martin, C. J., Gaffney, S., Greene, J. H., \& Ikizler, T. A. (2005). Physical activity patterns in chronic hemodialysis patients: Comparison of dialysis and nondialysis days. Journal of Renal Nutrition, 15(2), 217-224. doi: 10.1016/J.Jm.2004.08.002

Matsuzawa, R., Matsunaga, A., Wang, G. Q., Kutsuna, T., Ishii, A., Abe, Y., ... Takahira, N. (2012). Habitual physical activity measured by accelerometer and survival in maintenance hemodialysis patients. Clinical Journal of the American Society of Nephrology, 7(12), 2010-2016.

Mercer, T. H., Crawford, C., Gleeson, N. P., \& Naish, P. F. (2002). Low-volume exercise rehabilitation improves functional capacity and selfreported functional status of dialysis patients. American Journal of Physical Medicine \& Rehabilitation, 81(3), 162-167. doi: 10.1097/00002060200203000-00002

Odden, M. C. (2010). Physical functioning in elderly persons with kidney disease. Advances in Chronic Kidney Disease, 17(4), 348-357. doi: 10.1053/ j.ackd.2010.02.002

Painter, P. (2005). Physical functioning in end-stage renal disease patients: Update 2005. Hemodialysis International, 9(3), 218-235.

Painter, P., Carlson, L., Carey, S., Paul, S. M., \& Myll, J. (2000a). Low-functioning hemodialysis patients improve with exercise training. American Journal of Kidney Diseases, 36 (3), 600-608.

Painter, P., Carlson, L., Carey, S., Paul, S. M., \& Myll, J. (2000b). Physical functioning and health-related quality-of-life changes with exercise training in hemodialysis patients. American
Journal of Kidney Diseases, 35(3), 482-492. doi: 10.1016/S0272-6386(00)70202-2

Painter, P., Ward, K., \& Nelson, R. D. (2011). Selfreported physical activity in patients with end stage renal disease. Nephrology Nursing Journal, $38(2), 139-147$.

Sasaki, J., John, D., \& Freedson, P. (2011). Validation and comparison of ActiGraph activity monitors. Journal of Science and Medicine in Sport, 14, 411-416. doi: 10.1016/j.jsams.2011.04.003

Segura-Orti, E., \& Johansen, K. L. (2010). Exercise in end-stage renal disease. Seminars in Dialysis, 23(4), 422-430. doi: 10.1111/j.1525-139X.20 10.00766.x

Stack, A. G., Molony, D. A., Rives, T., Tyson, J., \& Murthy, B. V. (2005). Association of physical activity with mortality in the US dialysis population. American Journal of Kidney Diseases, 45(4), 690-701. doi: 10.1053/j.ajkd.2004.12.013

Storer, T. W., Casaburi, R., Sawelson, S., \& Kopple, J. D. (2005). Endurance exercise training during haemodialysis improves strength, power, fatigability and physical performance in maintenance haemodialysis patients. Nephrology, Dialysis, Transplantation, 20(7), 1429-1437. doi: $10.1093 /$ ndt/gfh784

Tamura, M. K., Covinsky, K. E., Chertow, G. M., Yaffe, K., Landefeld, C. S., \& McCulloch, C. E. (2009). Functional status of elderly adults before and after initiation of dialysis. New England Journal of Medicine, 361(16), 1539-1547. doi: 10.1056/NEJMoa0904655

Troiano, R. P., Berrigan, D., Dodd, K. W., Masse, L. C., Tilert, T., \& Mcdowell, M. (2008). Physical activity in the United States measured by accelerometer. Medicine and Science in Sports and Exercise, 40(1), 181-188. doi: 10.1249/mss.0b013e3 $1815 \mathrm{a} 51 \mathrm{~b} 3$

United States Renal Data System (2012). Annual Data Report: Atlas of Chronic Kidney Disease and End-Stage Renal Disease in the United States. Disponível em: www.usrds.org/atlas.aspx

Van Heuvelen, M. J. G., Kempen, G. I. J. M., Ormel, J., \& Rispens, P. (1998). Physical fitness related to age and physical activity in older persons. Medicine and Science in Sports and Exercise, 30(3), 434-441. doi: 10.1097/00005768-19980300000015 